ORIGINAL STUDY

\title{
Symmetry patterning by the causative organism of rhinosporidiosis in culture
}

\author{
Dhara B. Dhaulakhandi', Alok K. Ravi', Lalit C. Garg', Karvita B. Ahluwalia' \\ ${ }^{1}$ Cell Biology and Electron Microscope Section, Department of Biophysics, All India Institute of Medical \\ Sciences, New Delhi, India \\ ${ }^{2}$ Gene Regulation Laboratory, National Institute of Immunology, New Delhi, India
}

\section{ABSTRACT}

Rhinosporidiosis is a chronic polypoidal infection of the nose, conjunctiva and other sites, believed to be caused by a fungus, Rhinosporidium Seeberi, with a doubtful taxonomy. Polyps contain histological round bodies and the exact mode of infection is not known. The round bodies are filled up with spherules. In tissue the organism forms spherical round bodies approaching 50 $500 \mu$ in diameter that contain innumerable single- celled organisms that mature at different rates. Mature organisms are approximately $7-9 \mu$ in size and escape through a pore that develops in the wall of the round body. The round body does not exist in nature outside the host.

The organism in rhinosporidiosis was believed to be uncultivable, until we cultured it for the first time in our laboratory. We further modified the culture medium and succeeded in culturing the causative agent of the disease in CBEML (Cell Biology and Electron Microcopy Laboratory) medium. Here we present some of the peculiar conspicuous features of the organism in culture leading to symmetry patterning.

KEYWORDS: rhinosporidiosis, polyps, nose, Rhinosporidium Seeberi, pattern

\section{INTRODUCTION}

Rhinosporidiosis is a polypoidal granulomatous disease affecting mainly the nasal septum and the inferior turbinates, the pharynx, the eyelids, and the skin (Figure 1A, B). The polyps are pink to purple, heavily vascularised and friable. They are chronic but not painful. Asymptomatic nodular skin lesions with multiple, discrete, sessile and lobulated or pedunculated, smooth and warty lobes of variable sizes and shapes are found.

Microscopic sections of polyps consistently display numerous round bodies filled with spherules considered as a diagnostic pattern for the disease (Figure 2A). Large-sized round bodies discharge the spherules through pores or breakdown of wall (Figure 2B). Released spherules are seen to enlarge and develop into new round bodies. This self-repeating cycle is accompanied by an increase in the size of polyps into massive polypoidal growths.

Rhinosporidiosis is not contagious, nor does it seem to be transmitted from animals to humans. Since case histories of most patients show bathing and dipping in ponds and natural water, most investigators believe in the aquatic origin of the organism in rhinosporidiosis. Though in recent years this disease has been a focus of several studies due to its prevailing etiologic controversies, many aspects of the disease and the causative agent (round body) have remained enigmatic to date. Different authors have presented varying degrees of arguments on the phylogenetic position of the causative agent of this disease.

There are many aspects of the disease and the causative pathogen remains problematic and enigmatic. These include: the pathogen's natural habitat, many aspects of its "life cycle", its immunology, cultural characteristics, and reasons for failure in the transmission of the causative organism in experimental animals, a hence paucity of information on its drug sensitivity. There is no cure for this disease and multiple recurrences are common after surgical excision. Antifungals, other chemotherapies and radiation are ineffective.

Rhinosporidiosis has been reported from many countries of the world, India and Sri Lanka being endemic for the disease. More than $88 \%$ of the reported cases in the world are from India and Sri Lanka ${ }^{1}$. The incidence of this disease is increasing and cases are being reported from North Indian states including 


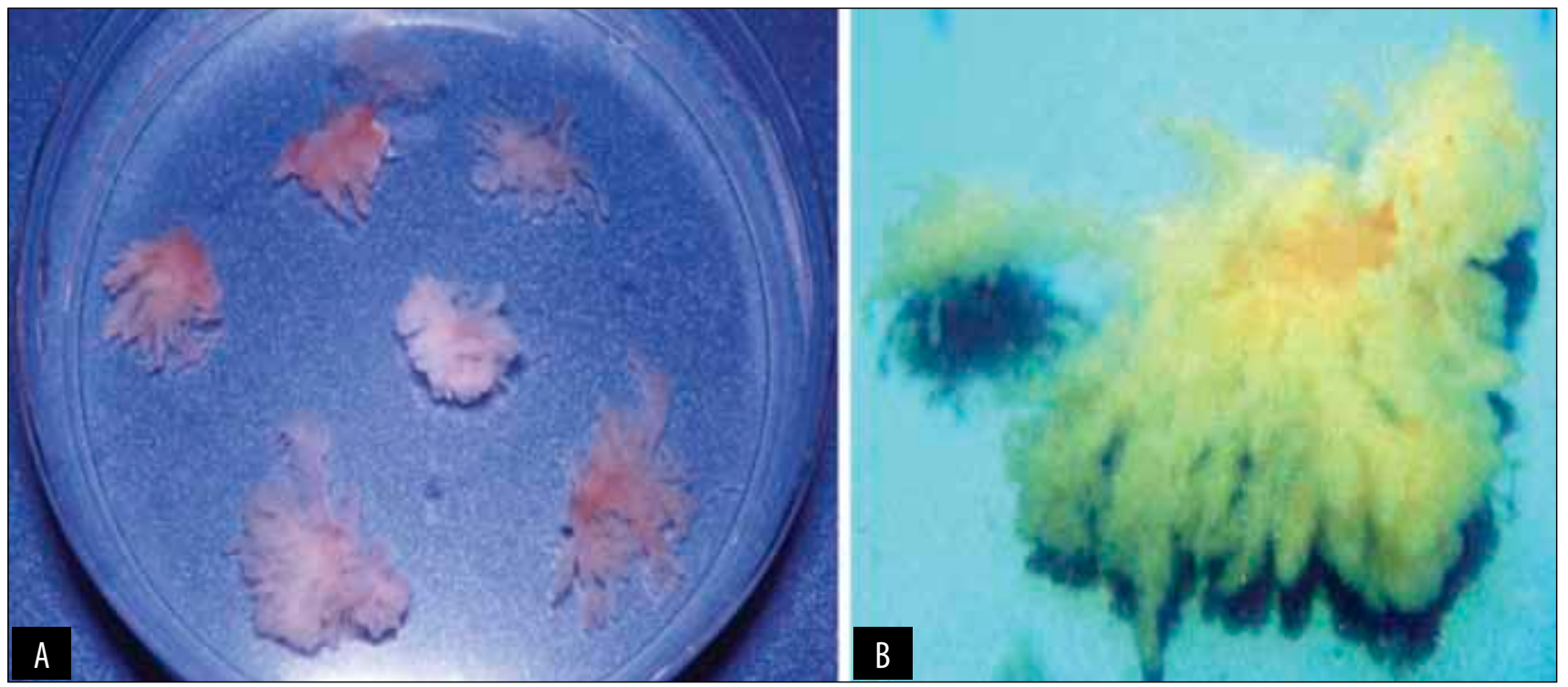

Figure 1 A. Portions of polypoidal mass in Petri dish showing ovulated morphology. B. Dot-like round bodies visible to naked eyes adhering to the surface of the polyp.

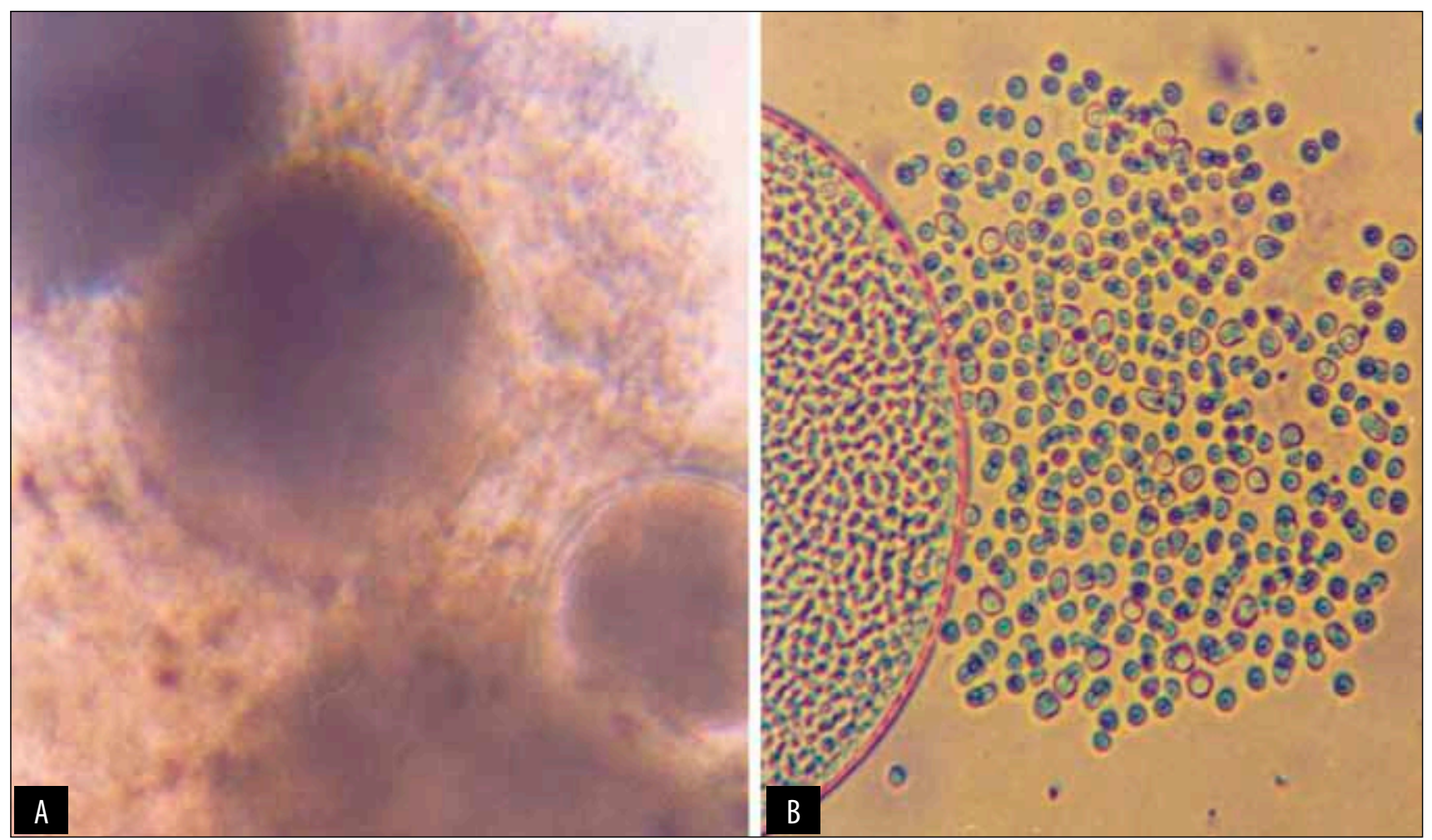

Figure 2 A. Light micrograph showing round bodies adhered to the polyp. B. Causative organisms of rhinosporidiosis released from a round body.

Delhi, where the disease was previously unknown. The incidence of the disease is higher in males than females.

We have previously published a method to culture the organism in rhinosporidiosis and have discussed some of its structural and cultural characteristics ${ }^{2}$. Extending our previous observations, here we report some unique features which have become available due to its growth on a modified culture medium ${ }^{3}$.

\section{MATERIAL AND METHODS}

Fresh surgically excised polyps of rhinosporidiosis were collected and transported from the operation theatre of All India Institute of Medial Sciences (AIIMS) and Safdarjung Hospital ENT Departments. The samples were collected in sterile $1 \mathrm{M}$ Tris ( $\mathrm{pH} 8.0$ ) or PBS ( $\mathrm{pH}$ 7.4). Samples were transported under sterile conditions to the Cell Biology and Electron Micros- 
copy Laboratory of the Department of Biophysics, AIIMS and processed for culturing in CBEML Medium developed by Dhaulakhandi et al (2006) ${ }^{3}$.

Polyps in patients with nasal rhinosporidiosis were observed as fleshy outgrowths of the mucous membrane (Figure 1A, B). The polypoidal growths appeared as pink to flesh coloured, oval, round or as lobulated masses. After surgery, the mass was dissected and examined for gross features and presence of round bodies. Dot-like round bodies varied in diameter from 100 to $400 \mu$, some adhering to the polyp surface, others floating with epithelial cells in the mucosal exudates (Figure 2A).

Three surgically excised samples of polypoidal masses of rhinosporidiosis collected in 1X PBS ( $\mathrm{pH}$ 8.0) were washed in sterile glass distilled water in order to remove RBCs and debris. The sample was then cut into about 20 roughly 1 to $2 \mathrm{~mm}^{2}$ pieces with the help of a sterile scalpel under laminar hood. Aseptic conditions in the culture room were checked by keeping a plain Amp-Agar plate and noting absence of growth. The pieces cut from the polyp were placed in a $50 \mathrm{ml}$ Falcon tube containing sterile liquid culture medium consisting of 2 Parts Salts-trace metals solution, $\mathrm{pH} 7.0\left(0.8 \% \mathrm{NaCl}, 0.02 \% \mathrm{KCl}, 0.02 \% \mathrm{CaCl}_{2}\right.$, $0.01 \% \mathrm{MgCl}_{2}, 0.2 \% \mathrm{NaH}_{2} \mathrm{PO}_{4}, 0.04 \% \mathrm{NaHCO}_{3}, 0.02 \%$ $\mathrm{K}_{2} \mathrm{HPO}_{4} \mathrm{H}_{3} \mathrm{BO}_{3}(2.86 \mathrm{mg} / \mathrm{l}), \mathrm{FeCl}_{3} 6 \mathrm{H}_{2} \mathrm{O}(0.3 \mathrm{mg} / \mathrm{l})$, $\mathrm{MnCl}_{2} .4 \mathrm{H}_{2} \mathrm{O}(0.81 \mathrm{mg} / \mathrm{l}), \mathrm{ZnSO}_{4} .7 \mathrm{H}_{2} \mathrm{O}(0.22 \mathrm{mg} / \mathrm{l})$, $\mathrm{Na}_{2} \mathrm{MoO}_{4} .2 \mathrm{H}_{2} \mathrm{O}(0.39 \mathrm{mg} / \mathrm{l}), \mathrm{CuSO}_{4} .5 \mathrm{H}_{2} \mathrm{O}(0.079$ $\left.\mathrm{mg} / \mathrm{l}), \mathrm{Co}\left(\mathrm{NO}_{3}\right)_{2} 6 \mathrm{H}_{2} \mathrm{O}(0.0494 \mathrm{mg} / \mathrm{l})\right), 1$ part $50 \mathrm{mM}$ PBS, $0.05 \%$ glycerol and cycloserine $(50 \mu \mathrm{g} / \mathrm{ml})$. Incubation of polyp pieces in this medium apparently reduces adhesion and facilitates the release of round bodies. Pieces of the polyp $\left(\sim 1 \mathrm{~mm}^{2}\right)$ were then shaken vigorously in a capped sterile Greiner tube containing 1X PBS for 20 minutes. Free round bodies (Figure 2A) were collected by slow-speed centrifugation $(500 \mathrm{rpm})$ or gravity sedimentation, and examined under Olympus (BX 50) light microscope. Round bodies were found to be completely free of adhering human material. Out of 120-150 round bodies isolated we selected a single round body of the largest size $(\sim 450-500 \mu)$. The selected single round body was placed on a clean glass side which had been previously washed with Teepol and distilled water and wiped with alcohol. This slide was briefly heat sterilised by alternatively placing and removing the slide onto the flame. Small glass chips of $1 \mathrm{~mm}^{2}$ were prepared and were also briefly heat sterilized, in the same way as the glass slide. A pure round body which was until now in sterile distilled water was placed on the glass slide and a glass chip was placed on it. A $200 \mu$ pipette tip was made blunt-ended (closed) with the help of the flame. With this blunt-ended pipette tip fitted to the micropipette a very gentle pressure was exerted by slowly moving the pipette tip in a zig-zag manner. The round body is ruptured in this process and cells are released. The glass slide supports the pressure exerted. The daughter cells isolated from the round bodies in-vivo were made to grow on CBEML medium under exposure to white fluorescent light of 2000 lx intensity on a Petri dish.

This study was carried out in accordance with the ethical standards as laid down in 1964's Declaration of Helsinki. All patients gave their informed consent for their inclusion in the study.

\section{RESULTS}

The daughter cells within the round bodies in vivo are surrounded by a glucomannan coat and contain conspicuous refractile granules. Daughter cells from round bodies were seen to organise dark brown macrocolonies on the culture medium. As observed under a laser confocal microscope, each daughter cell in a round body produces $6-8$ smaller cells termed nanocytes, which are liberated from the parent cell. Electron micrographs of a round body and daughter cells display various electron-dense cell inclusions, lipidic bodies, concentric laminated bodies, vacuoles, and membranous vesicles with angular profiles and dispersed chromatin.

The daughter cells isolated from round bodies in vivo were made to grow on the CBEML medium under exposure to white fluorescent light of $2000 \mathrm{~lx}$ intensity. Cells were seen to divide by binary fission (Figure 3A, B). Progeny cells were again seen to divide, thus increasing the number of cells. Figure $4 \mathrm{~B}$ shows four cells produced by binary fission in one plane followed by another binary division in a plane at right angles to the first plane. Some cells are seen to grow bigger and circular, bipartite structures and finally become macrocolonies as previously reported by us in slide culture?

In CBEML culture medium at many instances some cells tend to colonize and form biofilm-like structure (Figure 4). We had confirmed that these were not the aggregates of crystals in the culture medium by doing a control experiment where organisms in the culture were not inoculated, and no such colonies were observed. In three to four-month-old cultures, the organism tends to form crystals and forms bunch of grape-like structures (Figure 5A, B). Like our previous observations in slide culture, the organism also forms in CBEML medium anucleated filamentous structures (Figure 6).

Division in culture takes place by binary fission in one or more planes (Figure 7A, B). In culture, we have observed progressive stages of the peculiar two-dimensional linear branching pattern of division (Figure 7C$\mathrm{K})$. The branching in the first 48-72 hours of growth (after inoculation into CBEML medium) was less 


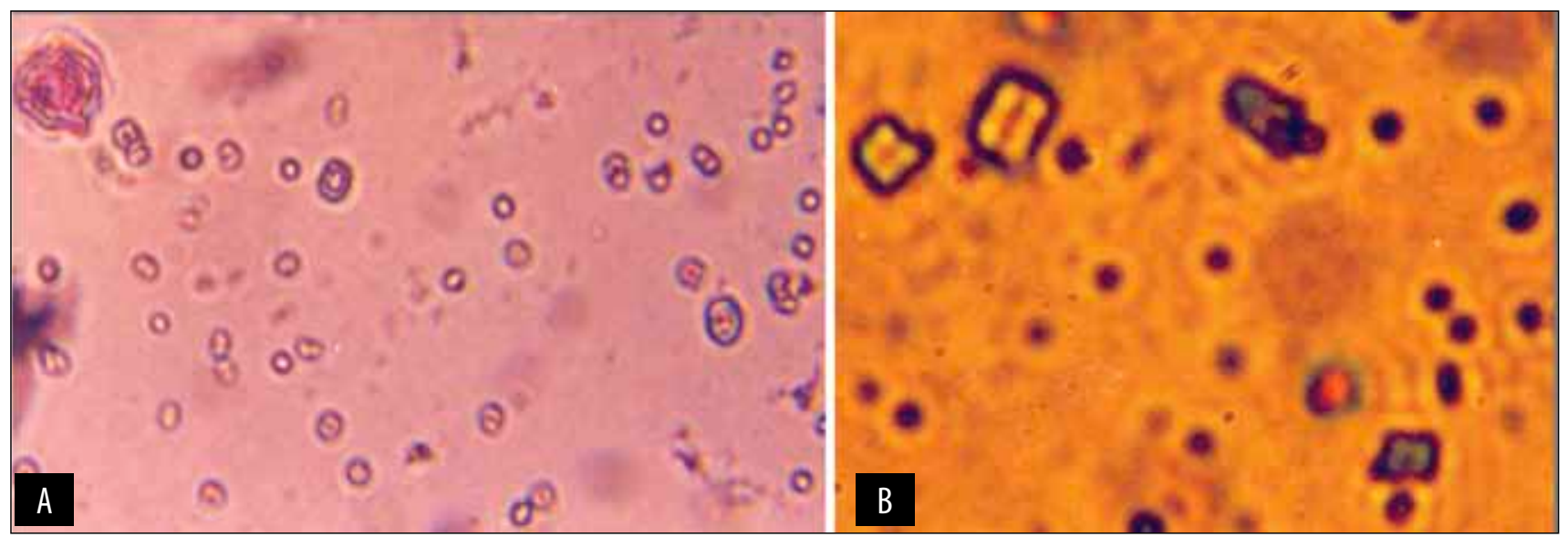

Figure 3 A. Dividing cells and some macrocolony-like structures in CBEML culture medium. B. Causative agent of rhinosporidiois showing division in more than one plane in CBEML culture medium.

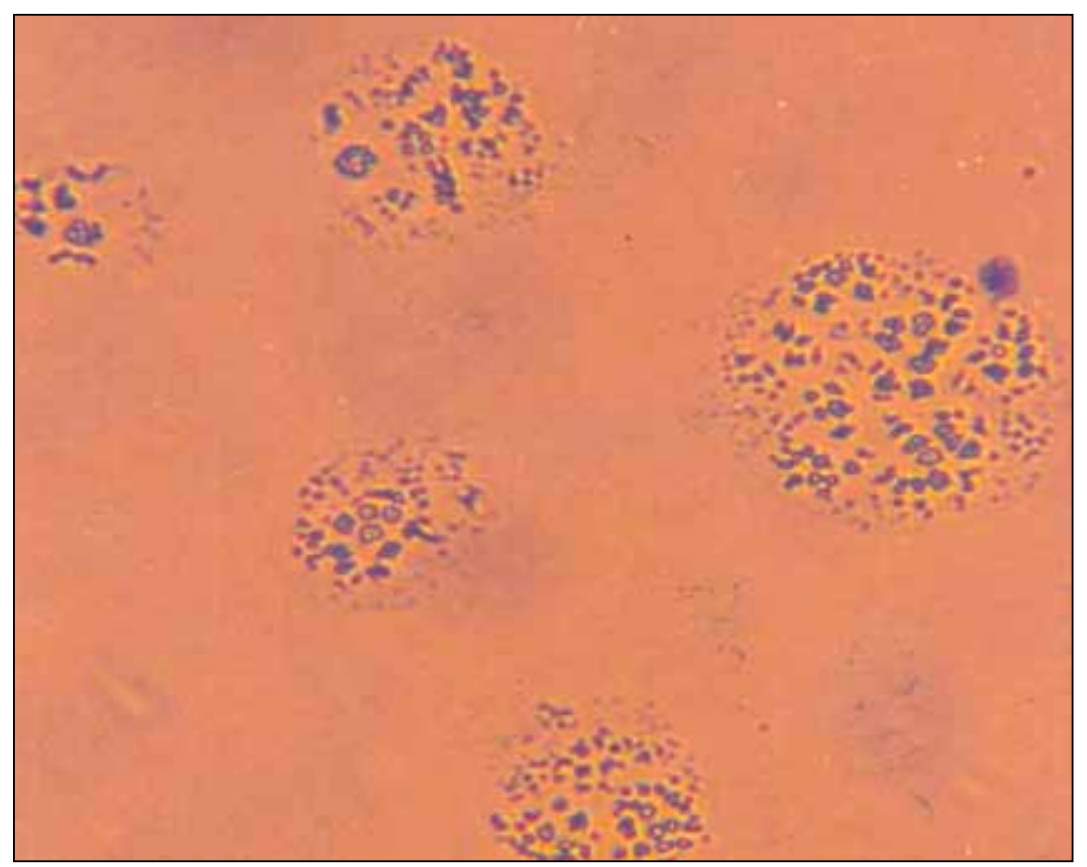

Figure 4 The causative agent of rhinosporidiosis forming a biofilm-like structure.

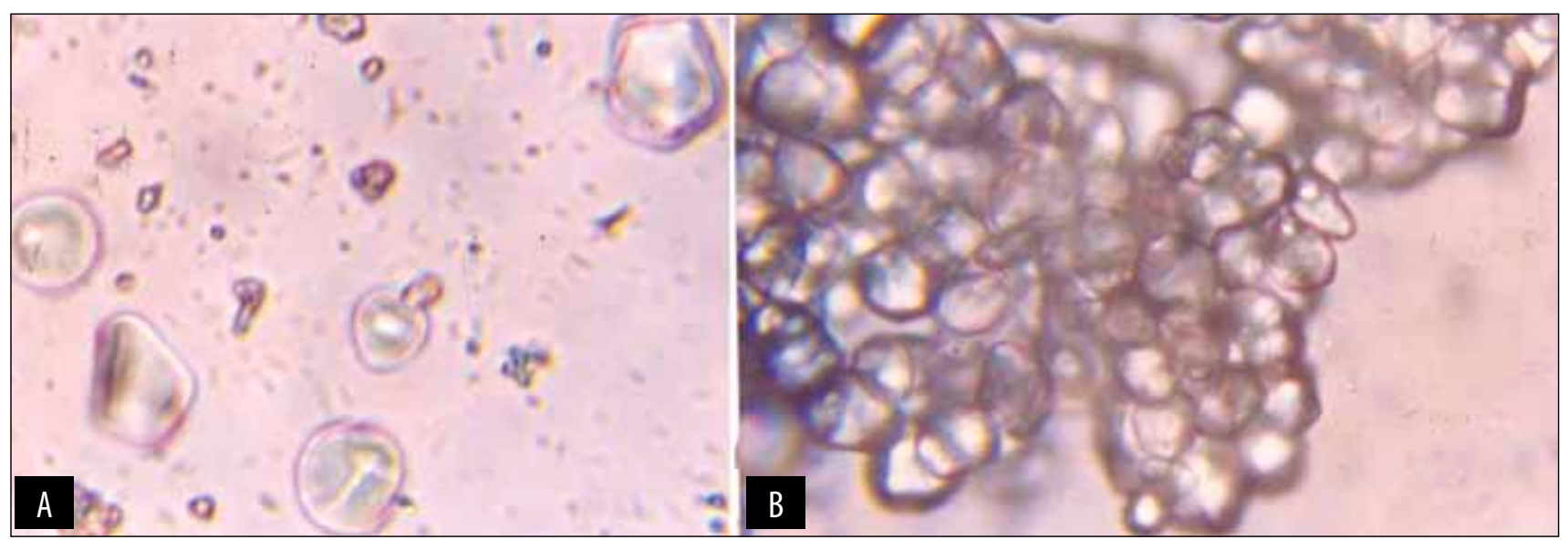

Figure 5 A. B. Crystal formation by the causative agent of rhinosporidiosis in CBEML culture medium. 


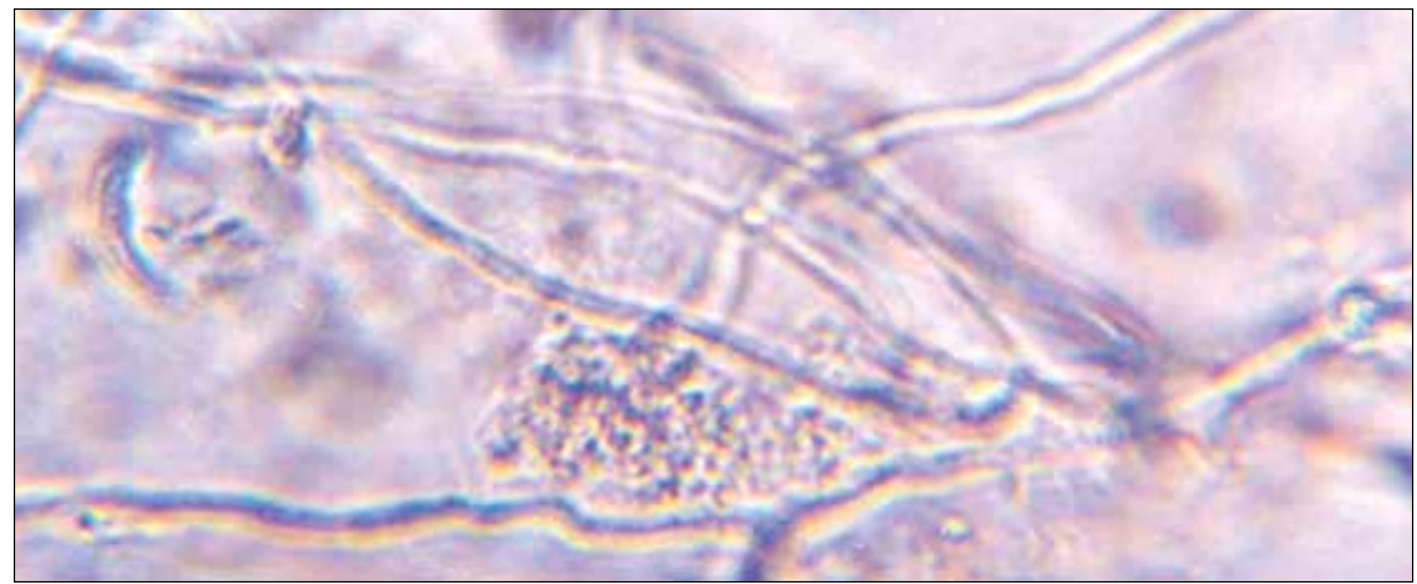

Figure 6 Anucleated filamentous structure formation by the causative agent of rhinosporidiosis in CBEML culture medium.

branched (mainly primary branching (Figures 7C to $7 \mathrm{H})$ ) compared to 48-72 hours of growth (beginning of secondary branching, Figure 7I). A very prominent secondary branching pattern was seen at the end of 96-120 hours giving it a necklace- or lace-like appearance (Figure 7G). Elaborate and magnificent tertiary and quaternary branching goes on with time with trivalent and pattern becoming evident after 3 to 4 months (Figure 7I-7K).

\section{DISCUSSIONS}

The causative agent of rhinosporidiosis presents many enigmatic features yet maintains symmetry. Starting from binary fission, it presents a complex symmetrical branching pattern. To study the molecular mechanism controlling the symmetry of the organism during the course of growth and the development in vivo and in vitro remains an important aspect of investigation. Probably the complex symmetrical branching might provide the organism the capability to survive in epithelial tissue in dark under anaerobic conditions. It is not unlikely that the causative agent of rhinosporidiosis after gaining entry to the host tissue might live in epithelial environment in a quiescent stage during unfavourable environment and may get activated when the right trigger for division and growth becomes available.

The published literature provides evidence that the growth patterns become more and more complex as the environmental conditions become less favourable. It is plausible to believe that the organism in rhinosporidiosis might tend to respond to adverse growth conditions by adopting more complex strategies and higher micro level organization in order to cooperate more efficiently. Beautiful patterns are formed by bacterial colonies under different environmental conditions. In bacteria, such strategies include differentiation and increase in length, production of extracellu- lar wetting fluid and secretion of surfactants that change the surface tension or chemotactic response to chemical agents. When grown on soft semi-solid substrates with high levels of nutrients, bacterial colonies grow in simple compact patterns. When a hostile nutritional environment is created using a very low level of nutrients in the laboratory in the Petri dish, very complex colonial patterns are formed. To counter hostile environmental conditions the bacteria develop sophisticated cooperative behaviour and intricate communication capabilities. Through such adaptive measures in response to adverse growth conditions, the colonies develop complex spatio-temporal patterns.

While working with Bacillus subtilis, in 1989, a Japanese group reported for the first time that bacterial colonies can grow elaborate fractal branching patterns ${ }^{4}$. This work was subsequently extended to other bacterial species as well such as Escherichia coli and Salmonella typhimurium ${ }^{5}$. Ben-Jacob et al. have extensively studied the adaptive behaviour leading to chiral and vortex forming morphotypes in bacteria ${ }^{6-13}$. With varying growth conditions, a profusion of patterns is exhibited by each morphotype. These alluring shapes and designs are part of the sophisticated strategies employed by the bacteria for cooperative self-organization in response to unfavourable growth conditions. Development of the morphotypes similar to bacteria may also be the likely adaptations for the causative agent of rhinosporidiosis surviving in light deficient environment in the nasal epithelium. It is also possible that such shapes could be the organizations so as to best utilize the space within the round bodies for efficient packaging, particularly for maintenance of the proliferation of single-celled organisms, which are born in innumerable number.

With myriad morphotypes evidenced from light, laser confocal and electron microscopy, the causative organism of rhinosporidiosis is likely to contain a sophisticated communication system with elevated 


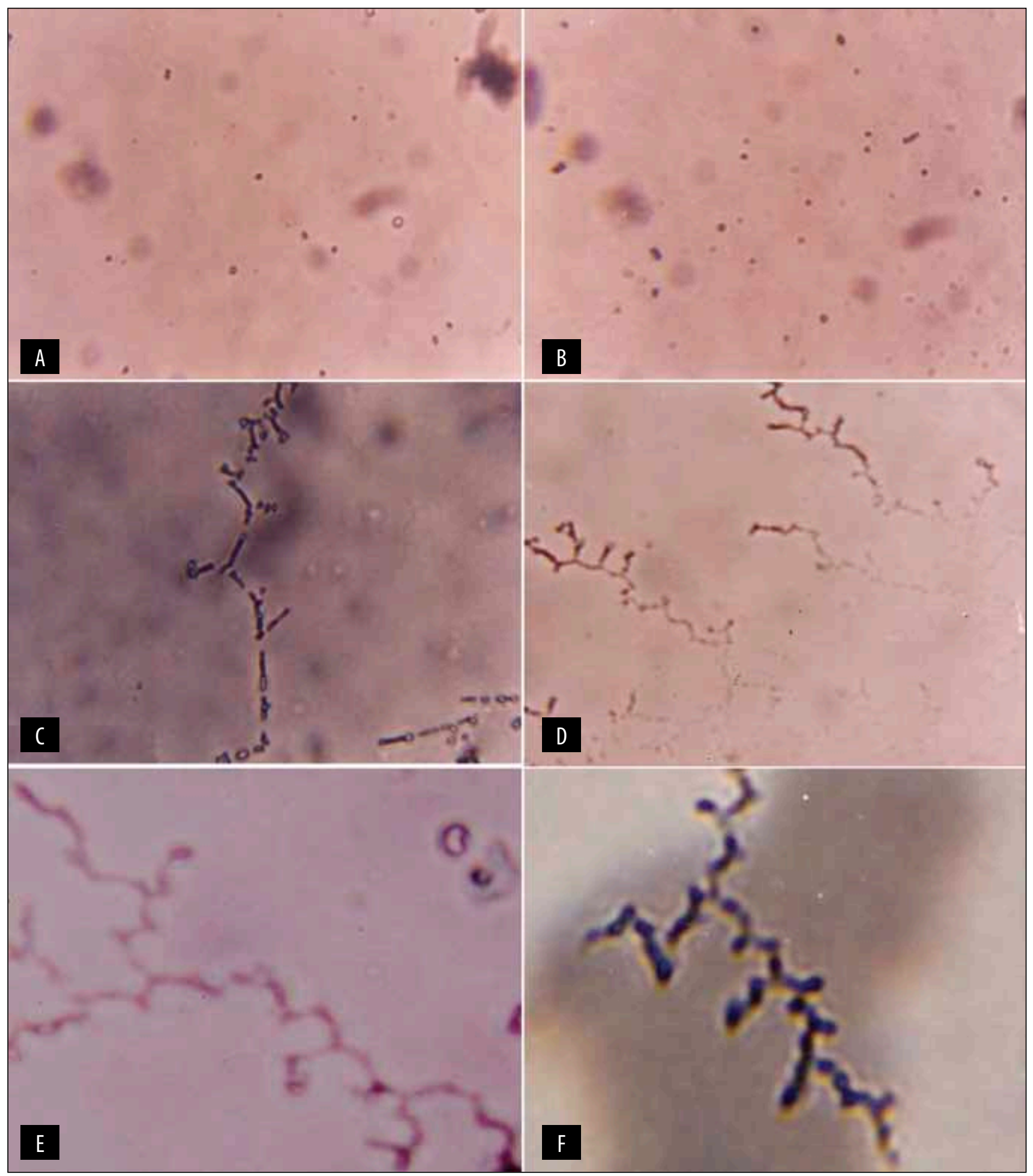

adaptability in terms of quorum sensing, chemotactic signalling and de novo gene formation. Accordingly, the colonial and branching pattern may be collectively self-engineered to encounter changing environmental conditions. The causative agent of rhinosporidiosis is seen to form biofilms typical to the bacteria. Biofilm represents a cell population encased in a hydrated matrix of exopolymeric substances, proteins, polysaccharides and nucleic acids. Biofilm formation in bacteria involves attachment and immobilization on a surface, cell-to-cell interaction, microcolony formation, and development of a three-dimensional biofilm structure. It needs to be investigated if the genes responsible for biofilm formation in the bacteria and the causative agent of rhinosporidiosis are the same or different. As some of the round bodies have been found without coat, it is not unlikely that the organism in rhinosporidiosis may afford to package without coat in a 


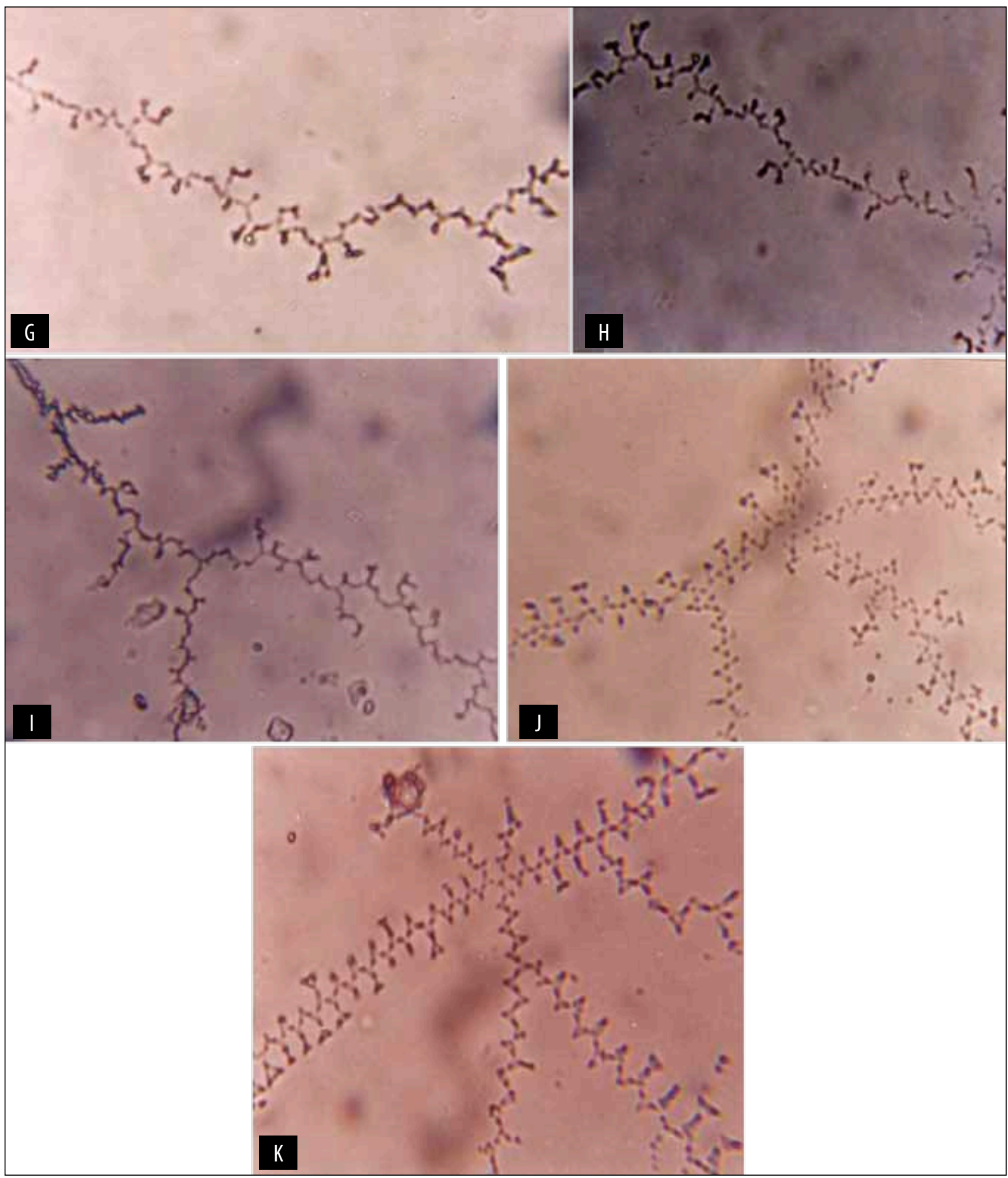

Figure 7 A. Day 0 of inoculation of the causative agent of rhinosporidiosis in the CBEML culture medium.

B. Day 1 of inoculation in the CBEML culture medium; some dividing cells and rod-like structures are visible.

C. Earliest stages of symmetry formation: liberated single-celled organism in CBEML medium (after 3 days of inoculation).

D. Symmetry formation in CBEML medium after 9 days of inoculation.

E. Symmetry formation in CBEML medium after 12 days of inoculation. F. Symmetry formation in CBEML medium after 15 days of inoculation.

G. Symmetry formation in CBEML medium after 18 days of inoculation.

H. Symmetry formation in CBEML medium after 21 days of inoculation.

I. Symmetry formation in CBEML medium after one month of inoculation.

J. Symmetry formation in CBEML medium after two months of inoculation.

K. Symmetry formation in CBEML medium after three months of inoculation. 
amoeboid structure with increasing susceptibility for spread of infection, thus helping in forming a polypoidal structure. Our studies in culture demonstrating biofilm-like structure further hints if the coat of the round body can be replaced at some stage by a thin film as the requirement of the organism in nasal epithelium. The genetic regulation of such biofilms and pattern formations in the causative agent of rhinosporidiosis emerges as a surprising intellectual mystery encountered never before.

The genetic control of self-assembly and branched patterning is not known at present, but it is believed that the evolving cellular pattern in different round bodies of various stages of maturity and development may assume newly cogenerated traits and abilities that are not explicitly stored in the genetic information of individual daughter cells. The peculiar branching pattern provides impetus to envisage that there might be genetic and epigenetic differences in the cells inside round bodies in various stages of development, as well as variation in exposure to diverse environmental conditions from patient to patient. The continuance of patterning in dark and in vivo in nasal epithelium cannot be ruled out. Maturation of the organism to a stage of rupture of the wall of the round body to release likely pathogenic cells, their penetration and internalisation into intracellular lacunae in the epithelial lining and further development to round bodies of evolving stages, seems to be a systematic and precise event. The single-celled organism in the round body and its conspicuous behaviour remains to be addressed from a geometrical, physical and chemical origin of form and symmetry point of view. It certainly seems to be operating at the level of the complex system where pattern formation, self-organization and organismal evolution are intriguing phenomena. Self-organization and patterning in the organism causative of rhinosporidiosis might require some kind of cell-cell interaction, signal transduction and system biology organization and coordination. Which genes and gene regulatory networks are responsible for such spatio-temporal dynamics of patterning remains an interesting aspect of the biology of rhinosporidiosis. An ensemble approach may be helpful to model systems capable of further advancing knowledge base in organismal and evolutionary biology of rhinosporidiosis.

\section{CONCLUSIONS}

The causative organism of rhinosporidiosis presents many enigmatic features including conspicuous sym- metry patterning. This may be a part of cooperative self-organization to cope with hostile environmental conditions. The single-celled organism in the round body and its conspicuous behaviour remains to be addressed from the view point of geometrical, physicchemical and genetic regulatory origins of form and symmetry. To the best of our knowledge, such symmetry formation by the causative agent of rhinosporidisois is being reported for the first time.

Conflict of interest: The authors have no conflict of interest.

Contribution of authors: All authors have equally contributed to this work.

\section{REFERENCES}

1. Kwon-Chung K.J., Bennett J.E. - Infections due to miscellaneous molds. In: Kwon-Chung K.J., Bennett J.E. (ed.) - Medical mycology. Lea \& Febiger, Malvern, Pa, 1992;pp.733-767.

2. Ahluwalia K.B. - Culture of the organism that causes rhinosporidiosis. J Laryngol Otol., 1999;113(6):523-528.

3. Dhaulakhandi D.B., Ahluwalia K.B., Ravi A.K., Garg L.C. - Detection of 16S rRNA gene in round bodies isolated from polyps of rhinosporidiosis. Infect Genet Evol., 2006;6(4):331-336.

4. Fujikawa H., Matsushita M. - Fractal growth of Bacillus subtilis on agar plates. J Phys Soc Jpn., 1989;58:3875-3878.

5. Matsuyama T., Matsushita M. - Morphogenesis by bacterial cells. In: Iannaccone P.M., Khokha M.K., editors - Farctal Geometry in Biological Systems, an Analytical Approach. CRC Press, New-York, 1995;pp.127-171.

6. Ben-Jacob E., Schochet O., Tenenbaum A., Cohen I., Czirok A., Vicsek T. - Generic modelling of cooperative growth patterns in bacterial colonies. Nature, 1994;368:46-49. doi: 10.1038/368046a0.

7. Ben-Jacob E. - Bacterial wisdom, Gödel's theorem and creative genomic webs. Physica A: Statistical Mechanics and its Applications, 1998;248(12):57-76

8. Ben-Jacob E., Levine H. - The artistry of microorganisms. Sci. Am., 1998b;279:82-87.

9. Ben-Jacob E., Cohen I., Gutnick D.L. - Cooperative organization of bacterial colonies: from genotype to morphotype. Annu Rev Microbiol., 1998c;52:779-806.

10. Ben Jacob E., Cohen I., Levine H. - Cooperative self-organization of microorganism. Adv Phys., 2000; 49(4):395-554.

11. Ben-Jacob E. - Bacterial self-organization: co-enhancement of complexification and adaptability in a dynamic environment. Philos Trans A Math Phys Eng Sci., 2003;361(1807):1283-1312.

12. Ben Jacob E., Becker I., Shapira Y., Levine H. - Bacterial linguistic communication and social intelligence. Trends Microbiol., 2004;12(8):366372.

13. Ben Jacob E., Aharonov Y., Shapira Y. - Bacteria harnessing complexity. J Biofilm., 2005;1(4):239-263. 